MKG-Chirurg 2017 $\cdot 10: 169-171$

DOI 10.1007/s12285-017-0116-3

Online publiziert: 30. August 2017

(c) Springer Medizin Verlag GmbH 2017

CrossMark

\author{
N.-C. Gellrich ${ }^{1}$ H. Essig ${ }^{2}$ A. Schramm ${ }^{3,4}$ \\ ${ }^{1}$ Klinik für Mund-, Kiefer- und Gesichtschirurgie, Medizinische Hochschule Hannover, Hannover, \\ Deutschland \\ ${ }^{2}$ Klinik für Mund-, Kiefer- und Gesichtschirurgie, Universitätsspital Zürich, Zürich, Schweiz \\ ${ }^{3}$ Klinik für Mund-, Kiefer- und Gesichtschirurgie, Universitätsklinik UIm, UIm, Deutschland \\ ${ }^{4}$ Klinik für Mund-, Kiefer- und Plastische Gesichtschirurgie, Bundeswehrkrankenhaus Ulm, Ulm, \\ Deutschland
}

\title{
Computerassistierte Planung und Chirurgie in der Mund-, Kiefer- und Gesichtschirurgie
}

Die moderne Medizin bietet durch den Einzug der Computertechnik neuartige Möglichkeiten, sog. Volumendatensätze aus Computer-, Magnetresonanz- oder Digitalen Volumentomographen über die reine medizinische Befundbeschreibung hinaus weiterzuverwenden. Dieses Weiterverwenden besteht beispielsweise darin, dass verschiedene Bilddatensätze, die zu verschiedenen Zeitpunkten erhoben worden sind, übereinandergelegt, d. h. fusioniert, werden können und dass virtuell in einem Datensatz Zielstrukturen markiert oder auch virtuelle Operationsergebnisse erstellt werden können. Um bereits präoperativ solche Datensatzveränderungen durchzuführen, bedarf es moderner interaktiver Bildanalyseund Konstruktionssoftware.

Wichtig für den Patienten ist, dass zu keinem Zeitpunkt die ursprüngliche Bildinformation verloren geht, sondern $\mathrm{zu}$ jeder Zeit und beliebig oft wieder aufgerufen werden kann. Neu ist jedoch, dass solche Bilddatensätze Bestandteil eines digitalen Workflows sind, sodass laufende Behandlungen in ihren Schritten qualitätsgesichert durchgeführt werden können.

In der Mund-, Kiefer- und Gesichtschirurgie (MKG-Chirurgie) ist dies bereits in mehreren Bereichen Routine. Vorteil des Fachs ist, dass viele Operationsplanungen knochenbasiert geplant werden können - selbstverständlich unter genauer klinischer Wertung der Weichgewebe. Hier lassen sich solche
Datensätze allein oder kombiniert bei der Behandlung angeborener oder erworbener Fehlbildungen/-stellungen (unfall-, tumor-, oder entzündungsbedingt) nutzen.

Die computerassistierte Planung hat dabei das Ziel, zusätzlich zu den bislang etablierten Behandlungsmethoden einen weiteren Qualitätssicherungsschritt, z. B. während einer Operation durch die Verwendung von intraoperativer Navigation oder Bildgebung, einzubringen. Zudem besteht die Möglichkeit, im Hinblick auf eine Individualisierung der Therapie beispielsweise patientenspezifische Ersatzstrukturen und Implantate bereits vor einer Operation herzustellen. Des Weiteren kann durch die interaktive Verwendung von Bildinformation eine Optimierung der interdisziplinären Behandlung, insbesondere bei Patienten mit Tumorerkrankungen oder nach Polytrauma, erzielt werden: Verschiedene Fachbereiche können alle auf dieselbe Bildinformation zugreifen. Hierbei ist die interaktive Verwendung von Bildinformationen nicht nur wesentlicher Bestandteil für die erfolgreiche Behandlung, z. B. im Rahmen der Entfernung eines Tumors, sondern sie kann auch weiter genutzt werden, um eine weitestgehend originalgetreue Wiederherstellung und damit eine möglichst hohe Lebensqualität zu erreichen. Wichtig für den Patienten ist, dass die so optimierte Ausnutzung von Bildinformationen keinerlei zusätzliche Strahlenbelastung bedeutet, sondern dazu dient, 
die individuelle Behandlung nachhaltig $\mathrm{zu}$ verbessern.

Die MKG-Chirurgie hat sich bereits seit Jahren spezialisiert, um die computerassistierte Planung und Chirurgie weiterzuentwickeln und nun auch durch weitere Elemente, wie z. B. die Interaktion mit der Biomedizintechnik zur Erstellung patientenspezifischer Implantate im Rahmen der Individualisierung der Medizin, zu ergänzen. Hierdurch ergibt sich die logische Ergänzung in Richtung Einsatz von Computerassistenz da, wo sie klinisch sinnvoll ist.

Für die Patienten ist wesentlich, dass Computerassistenz lediglich eine Ergänzung der klinischen Erfahrung und keinerlei Ersatz dafür ist. In den folgenden Beiträgen erläutern Experten den möglichen Einsatz von Computerassistenz.

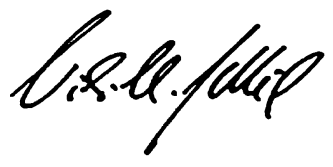

Univ.-Prof. Dr. Dr. N.-C. Gellrich

\section{Korrespondenzadresse}

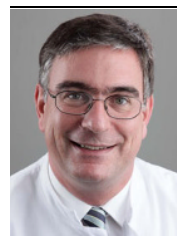

Univ.-Prof. Dr. Dr.

N.-C. Gellrich

Klinik für Mund-, Kiefer-

und Gesichtschirurgie,

Medizinische Hochschule

Hannover

Carl-Neuberg-Str. 1,

30625 Hannover,

Deutschland

Gellrich.Nils-Claudius@

mh-hannover.de

Interessenkonflikt. N.-C. Gellrich, H. Essig und A. Schramm geben an, dass kein Interessenkonflikt besteht.

\section{Erste Online-Krebskarte}

Mehr als 760 Onkogene haben Forscher des Broad Institute des MIT und von Harvard identifiziert und eine Krebskarte erstellt. In dieser Übersicht können Wissenschaftler jedes einzelne Gen aufrufen und herausfinden, bei welcher Tumorart in Abhängigkeit von welchen anderen Genen es agiert.

Bei der Analyse stellte sich heraus, dass 10\% der gelisteten Onkogene in vielen Krebsarten parallel eine tragende Rolle spielen. Dazu zählen etwa MDM4, PIK3CA, KRAS, IGF1R, CDK4, erklärt die Koautorin Francisca Vazquez vom Dana-Farber Cancer Institute in Cambridge. Therapien, die diese Krebsgene stilllegen, könnten daher bei mehr Krebspatienten zum Einsatz kommen. Nur bei einem Fünftel der Interaktionen zwischen den Onkogenen waren Genabschnitte involviert, die Forscher bereits zuvor für potenzielle Therapien ins Auge gefasst hatten. $90 \%$ der etwa 500 untersuchten Zelllinien waren zudem nur von mindestens einem Gen eines 76-Gensets stark abhängig. Daraus folgern die Forscher, dass viele Krebsarten sich auf relativ wenige Gene verlassen, um ihr Überleben zu sichern.

\section{Mehr als 17.000 Gene untersucht}

Ihre Versuche haben Aviad Tsherniak und seine Kollegen an etwa 500 Zelllinien durchgeführt, die mehr als 20 Krebsarten repräsentieren. Mit der RNA-Interferenz-Technik wurden mehr als 17.000 Gene stillgelegt, um zu untersuchen, welche Gene für die Krebszelle überlebenswichtig sind. Starben die Zellen ab, nachdem ein bestimmtes Gen via small interfering RNA (siRNA) abgeschalten wurde, bedeute das etwa, dass dieses Gen für die Zellteilung entscheidend war, erklärt David Root, Direktor der Genetic Pertubation Platform des Broad Cancer Programs.

\section{Onlinesuche für Onkogene möglich}

Wurde durch siRNA ein entscheidendes Onkogen getroffen, kompensiert die Krebszelle den Ausfall häufig, um zu überleben, indem andere Gene aktiviert werden. Diese Abhängigkeit zwischen Krebsgenen übertrugen die Forscher ebenfalls in ihre Krebskarte, die auch online abrufbar ist. Hier kann jedes Gen in eine Suchmaske eingegeben werden, um Abhängigkeiten zu überprüfen.

Mithilfe von Biomarkern untersuchten die Forscher auch, wie die Gene sich in Krebszellen veränderten. Überrascht waren sie, als sie entdeckten, dass sich mehr als $80 \%$ anpassten, indem sie die Expression eines Gens hoch oder herunter regulierten. Das passiert etwa durch Hypermethylierung eines Genabschnitts. Mutationen in der Gensequenz waren hingegen nur in $16 \%$ der Fälle relevant.
Quelle: Deutsches Ärzteblatt (www.aerzteblatt.de) basierend auf: Cell (2017) doi: 10.1016/j.cell.2017.06.010 
Hier steht eine Anzeige.

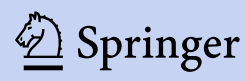

